\title{
Development of a Massively Parallel Parachute Performance Prediction Code
}

\author{
Carl W. Peterson, James H. Strickland, Walter P. Wolfe, W. David Sundberg, and Donald D. McBride \\ Unsteady \& Reactive Fluid Mechanics Department \\ Sandia National Laboratories \\ Albuquerque, NM
}

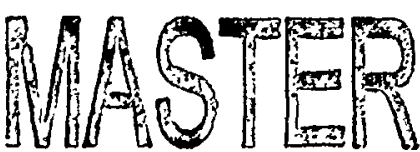

\section{$\underline{\text { Abstract }}$}

Sandia National Laboratories is initiating development of a complete numerical simulation of parachute performance, beginning with parachute deployment and continuing through inflation and steady state descent. The purpose of the parachute performance code is to predict the performance of stockpile weapon parachutes as these parachutes continue to age well beyond their intended service life. A new massively parallel computer will provide unprecedented speed and memory for solving this complex problem, and new software will be written to treat the coupled fluid, structure and trajectory calculations as part of a single code. Verification and validation experiments have been proposed to provide the necessary confidence in the computations.

\section{Introduction}

The Department of Energy has given Sandia full responsibility for the complete life cycle ("cradle to grave") of all nuclear weapon parachutes. This means that Sandia must have the capability to identify and respond to any anomalous performance results observed in flight tests of stockpile parachutes at any time during the remainder of the parachute systems' stockpile life. Recently, DOE mission planners have prescribed significant extensions of the life of the weapons in the stockpile. They have asked Sandia whether war reserve (WR) stockpile parachutes will be able to meet Stockpile-to-Target Sequence (STS) and Military Characteristics (MC) performance requirements in the future now that their intended stockpile life has been doubled from 20 years to $\sim 40$ years.

In order to answer this question, Sandia must (1) determine the effects of aging on parachute material strength and dimensions, and (2) determine how those changes affect the performance of the parachutes throughout the STS/MC parachute release conditions. We believe that our ongoing parachute material aging program allows us to assess the effects of aging on parachute material properties. However, Sandia presently has no science-based predictive tool for incorporating our growing knowledge of parachute material aging effects into a believable estimate of parachute performance at any time during the rest of its stockpile life. Our Joint Test Assembly (JTA) flight tests can assess today's performance of WR parachutes at the tested release condition, but they are not able to predict how WR parachutes will perform in the future, over the full range of STS/MC release conditions. Neither is the experience of experts able to predict future parachute performance with the requisite confidence.

While we agree that any capability for predicting future stockpile parachute performance will still require testing and would greatly benefit from access to knowledgeable experts, we remain convinced that only a modeling and simulation-based approach can provide us with a parachute performance predictive capability. Unfortunately, our existing computers cannot accommodate the detailed models of the coupled fluid and structural dynamics or the changing boundary conditions needed to simulate (with high confidence) future WR parachute performance. We need more powerful computational capabilities and improved numerical methods in order to solve this problem.

DOE established the Accelerated Strategic Computing Initiative (ASCI) because it recognized the need to provide future weapon system engineers and component designers with robust, believable predictive tools for stewardship of the nuclear weapon stockpile. The purpose of the ASCI Para-

This paper is declared a work of the U.S. Government and is not subject to copywrite protection in the United States, 


\section{DISCLAIMER}

This report was prepared as an account of work sponsored by an agency of the United States Government. Neither the United States Government nor any agency thereof, nor any of their employees, make any warranty, express or implied, or assumes any legal liability or responsibility for the accuracy, completeness, or usefulness of any information, apparatus, product, or process disclosed, or represents that its use would not infringe privately owned rights. Reference herein to any specific commercial product, process, or service by trade name, trademark, manufacturer, or otherwise does not necessarily constitute or imply its endorsement, recommendation, or favoring by the United States Government or any agency thereof. The views and opinions of authors expressed herein do not necessarily state or reflect those of the United States Government or any agency thereof. 


\section{DISCLAMMER}

Portions of this document may be illegible in electronic image products. Images are produced from the best available original document. 
chute Performance Code program is to accelerate the development of a numerical simulation capability for predicting the future performance of the nuclear weapon parachutes. The code must predict parachute deployment, inflation, time-dependent stresses and motion, and bomb/ parachute trajectories over the full range of $\mathrm{MC}$ and STS use environment and release conditions. It must include a detailed, ribbon-by-ribbon model of the parachute configuration and material properties in order to predict the effects of long-term aging on parachute performance.

No one in the world is close to having such a code. Many people do not believe that such a code will ever be created, let alone trusted. Sandia recognizes the magnitude of the challenge and acknowledges the risks. We believe that the ASCI Parachute Performance Code is a necessary (but not sufficient) tool for being able to predict parachute performance with the requisite fidelity to make stockpile parachute retain or replace decisions in advance of encountering a problem in a JTA test.

\section{Massively Parallel Computer Architecture}

Sandia has teamed with Intel Corporation to construct a new massively parallel computer of unprecedented performance. When completed in June 1997 , this computer will have 4536 pairs of commercially-available Intel Pentium Pro microprocessors operating in parallel, 600 gigabytes $\left(600 \times 10^{9}\right)$ of memory, and two terabytes $(2 \times$ $10^{12}$ ) of disc space. With new algorithms to balance the loads on each processor pair, this computer will be capable of achieving $1.8 \times 10^{12}$ floating point operations per second (1.8 teraFLOPs) running production code. Roughly three-quarters of the processors are already operating on site; benchmark calculations on that configuration show that this "teraFLOP computer" will have the power of between 2000 and 4000 Cray YMP computers.

\section{Computational Model of Parachute Performance}

Numerical Approach

Previous investigators and reviewers discuss the fluid dynamic methods for calculating parachute inflation and aerodynamics using both grid-based ${ }^{1-7}$ and gridless ${ }^{1,8-11}$ computational methods. They have identified strengths and weaknesses of each method relative to its ability to replicate the unsteady, porous, deformable bluff body fluid mechanics that drives ribbon parachute inflation. We must decide which approach should be used for this Parachute Performance Code implementation.

Selection of the approach to model the fluid dynamics code was determined by the physical time and spatial domains of parachute deployment, inflation and descent. The parachute and bomb travel approximately a quarter of a mile during a high-speed laydown delivery. The volume of air set in motion by parachute deployment and inflation is roughly $10^{12}$ cubic centimeters. Traditional gridbased computational methods would have to keep track of the air velocities in each cubic centimeter during the 4-second flight of the parachute, with new velocity calculations required approximately every 10 milliseconds. Even with adaptive grids and other advanced computing schemes, writing just the fluid dynamic velocity information would require storage of at least $10^{12}$ words.

The compute time required for a grid-based approach to the parachute performance problem is also unacceptably large. A grid-based calculation of a steady parachute flow field for a specified canopy shape at one time step took over 6 months of CPU time on a SPARCstation ${ }^{2}$; we estimate that this same "simplified" problem would take approximately an hour on Sandia's teraFLOP machine. With $\sim 400$ time steps per trajectory, the fluid dynamic computation alone (no coupling to the calculation of the evolving parachute shape or the canopy stresses) would take at least 400 hours to complete, excluding the time needed to regrid as the shape of the parachute changes.

Our assessment indicates that gridless vortex methods can significantly reduce memory and storage requirements and computing time compared to grid-based methods. Approximately $10^{8}$ vortex elements will be needed to simulate the 3-D unsteady flow field. Direct computation of the timedependent velocity field from the vorticity field using requires on the order of $\left(10^{8}\right)^{2}$ computations for an exact solution, but we expect our fast-solver 
implementation to reduce the number of computations to $O\left(\left[10^{8}\right] \times \ln \left[10^{8}\right]\right)$ at each time step.

We intend to couple Sandia's Vortex Method flow field solver (the axisymmetric version is called VPARA $^{9}$ ) to Sandia's large deformation finite element structural mechanics code (called PRONTO $^{12}$ ), which will compute the stresses and evolving shape of the parachute during inflation. PRONTO, which is already running on a massively parallel computer, will also be used to describe the kinematics of parachute deployment. The computational parachute configurations will be detailed kinematic, structural, and material models of weapon parachute geometries and will link to predictions and data describing all known effects of material aging. The simulations will predict performance over the full range of MC release velocities and altitudes, and STS temperature and storage/transport environments.

\section{VPARA Computational Fluid Dynamics Results}

One of the deficiencies of traditional vortex methods is that they do not account for the effects of viscosity. Many bluff body flows are not very sensitive to changes in Reynolds number and viscous effects can safely be ignored. We currently believe this to be the case for parachutes. However, as part of our general development of vortexbased computational methods, we are currently developing robust numerical algorithms that are compatible with vortex methods and account for the effects of fluid viscosity. Should our assumptions about the importance of viscosity in parachute aerodynamics prove incorrect, we can add these viscosity modules to our parachute inflation code at some future time.

Several attempts have been made in the past to couple the viscous diffusion process with Lagrangian vortex methods. Reviews of these attempts are contained in Refs. 13-15 and are not repeated here. The method we are developing uses the diffusion velocity concept. The diffusion velocity is defined such that the total circulation within a specified boundary remains constant if that boundary moves at the sum of the diffusion velocity and the local fluid velocity. The diffusion velocity equations, their derivation, and method of implementation are contained in Refs. 13-15. What we present here are comparisons of two-dimensional calculations using these methods for flows near walls, where vorticity is being generated and then convected and diffused away from the wall, with classical analytical solutions.

The first solution is the case for laminar flow over a finite length flat plate. This solution was generated by the code WALLYR which represents an exploratory implementation of the solution for wall layer flows. The solution was started with the plate moving to the left at velocity $U_{0}=1$. The results of the calculations for the vorticity profile at a Reynolds number based on the length from the plate's leading edge, $R e_{x}$, of $1 \times 10^{5}$ are shown in Figure 1 along with the exact solution given in Schlichting. ${ }^{16}$ Solutions at other Reynolds numbers and both the stream-wise and transverse velocity profiles also show excellent agreement with the exact solutions.

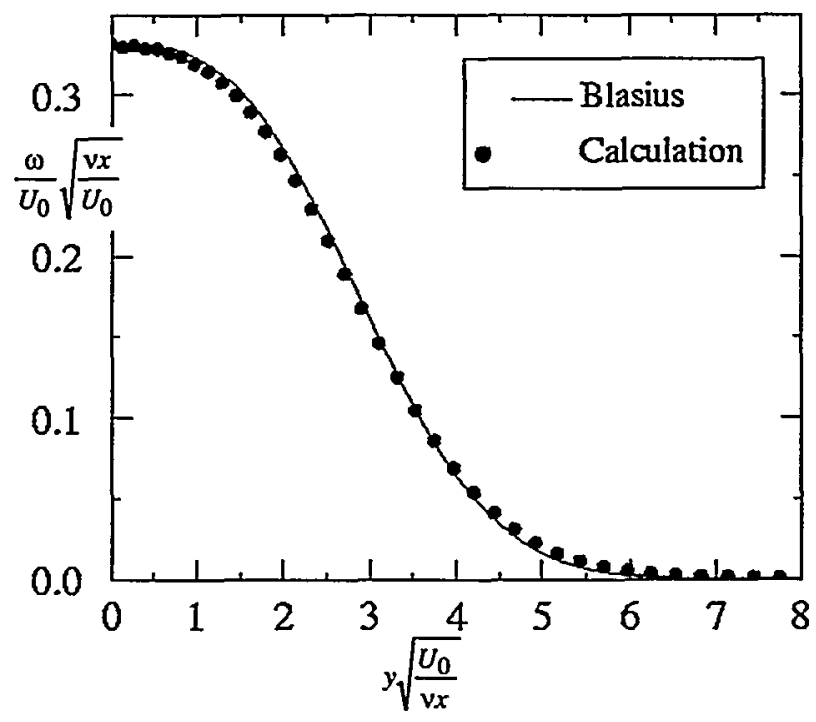

Figure 1. VPARA Laminar Flat Plate Vorticity Comparison with Blasius Solution

We next examine the case of a sinusoidally oscillating plate, which is sometimes referred to as Stokes Second Problem. ${ }^{17}$ After the plate has been oscillating for a few cycles, it reaches a periodic solution for vorticity $\omega$, given by

$$
\omega=s \cdot \exp (-s y)\{\cos (n t-s y)-\sin (n t-s y)\}
$$

where

$$
s=\{n / 2 v\}^{1 / 2}
$$


$y$ is the distance normal to wall, $t$ is time, $n$ is oscillation frequency in $\mathrm{rad} / \mathrm{sec}$, and $v$ is viscosity. Figure 2 shows the calculated and exact vorticity distributions for three angular displacements after completing five full cycles.

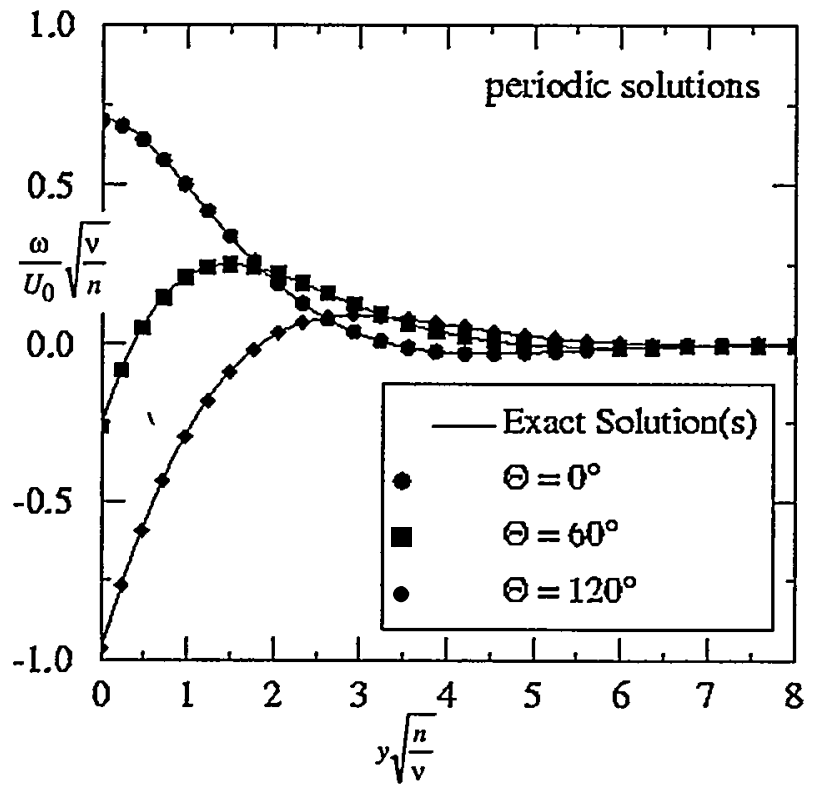

Figure 2. Comparison with Sinusoidally Oscillating Flat Plate

In addition to coupling the viscous diffusion process with Lagrangian vortex methods, FY97 efforts have focused on porting the VPARA code to the massively parallel computer. We have found that the fast solver method which works best with our vortex method on a single processor machine is difficult to parallelize on a massively parallel machine, so we are evaluating other fast solver methods to optimize computational performance on the teraFLOP computer. A simple structural code is being used instead of PRONTO to investigate the requirements for coupling between the fluid dynamic solution from the VPARA code and the equations that move the simulated parachute canopy. We intend to demonstrate an axisymmetric, incompressible, coupled fluid/structure massively parallel calculation of an axisymmetric parachute inflation by the end of FY97.

\section{Fluid/Structural Code Coupling}

The simplest coupling between a fluid and structural dynamics code for a parachute occurs by exchanging pressure and shape data at each time step. Initially, the body shape and velocity are specified. The fluids code then calculates the pressure distribution on the body surface. This pressure distribution is then passed to the structural dynamics code which calculates a new shape for the surface as well as new surface velocities. This information is then used by the fluids code to calculate a new pressure distribution. Unfortunately, this level of coupling has not been successful. For example, Stein, Benney, and Steeves ${ }^{4}$ found that they had to add artificially large "normal damping coefficients" to keep their calculations stable. Haug, Lasry, and Kermel ${ }^{7}$ reported on a benchmark simulation of parachute inflation which coupled ESI's PAM-FLOWTM and PAM-SOLIDTM codes. While no description of assumed damping coefficients were included in this paper, the resulting light-bulb shapes are not those typically seen in inflating parachutes.

Our recent investigations have suggested that the stability problem is related to the fact that the mass ratio associated with the parachute and the surrounding air tends to be small. The canopy/air mass ratio $M_{R}$ can be defined by:

$$
M_{R} \equiv \frac{m_{c}}{\rho D_{c}^{3}},
$$

where $m_{c}$ is the mass of the canopy, $\rho$ is the air density, and $D_{c}$ is the constructed diameter of the parachute. A typical value of $M_{R}$ for a weapons parachute is on the order of 0.03 . Thus the added mass of the air is large as compared to the mass of the canopy. If the added mass of the air is not explicitly represented in the structural dynamics code, the resistance to motion (acceleration) is relatively small since it is based only on the mass of the canopy. Thus, even though the fluid dynamics code calculates the correct pressures which include added mass effects, the structural dynamics code needs to be able to calculate the forces associated with acceleration of the fluid.

To illustrate the importance of placing the added mass terms explicitly into the dynamics model, a simple structural dynamics code was coupled with an empirically based fluid dynamics model to simulate parachute inflation. The simple structural code models the dynamics of only the suspension 
lines, radials, and vent lines, with all the canopy mass treated as if it were attached to the radial. The elastic effects of these components are included in the structural code. The empirical fluid dynamics model approximated the pressure forces as the sum of a static pressure term based on the current fraction of inflation, a damping term, the rate of change of a hypothesized distribution of apparent mass multiplied by its normal velocity change, and the normal acceleration of the apparent mass. When the fluid code was loosely coupled to the structural code (the apparent mass acceleration term was removed from the structural solution), the structural code was highly unstable. With explicit coupling of added mass terms in the dynamics model, the results are as shown in Figure 3. Clearly, the ability to model the response of the fluid to accelerations normal to the parachute surfaces within in the dynamics model is desirable.

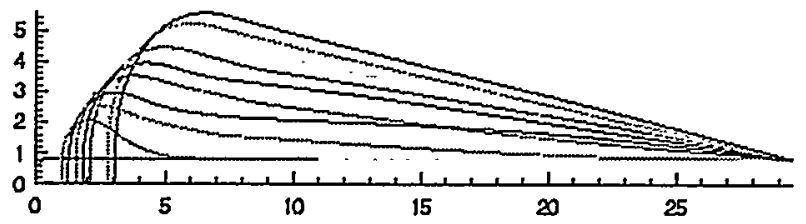

Figure 3. Canopy Shapes During Inflation, Including Apparent Mass Effects in the Canopy Shape Calculations

From this investigation, it is concluded that parachutes, being lightweight structures, will require a coupling of some of the fluid forces into the structural solution. The coupling is tighter than an alternating fluid-step then structure-step approach, but should not require simultaneous solution of both the fluid and structural equations in the solution process, if a proper apparent mass acceleration term can be identified and subtracted from the full fluid force and that apparent mass acceleration term included in the structural solution. By artificially making the parachute very massive, a non-temporal (but stable) solution can be obtained without an apparent mass acceleration term in the structural solution. Another conclusion is that an implicit structural solution method (with much larger time steps) can be used, with the fluid forces evaluated at the beginning of the time step and held constant for the time step (with the exception, of course, of the apparent mass acceleration term). Therefore, with an implicit solution, the time step can be governed by the explicit application of fluid forces. This means an implicit solution will only need to be concerned with change in structural forces, and therefore much simplified.

Moving forward with the coupling investigations, VPARA calculations have replaced the postulated fluid forces in the coupled fluid/ structure code. At the time of this writing, the equations to calculate apparent mass terms from VPARA have been determined and coding of them is just completed, but this matrix of apparent mass coefficients has not yet been incorporated into the structural code. Trials using VPARA for the pressure distribution have been conducted, but up until now they have required that an apparent mass acceleration term be hypothesized and included in the structural solution. With the apparent mass coefficients now available from VPARA, it doesn't warrant trying to refine a hypothesized term; instead, the calculated terms will be incorporated. Current experience with VPARA coupled to the structural code indicate that the total compute time for the explicit structural solutions is trivial compared to the total compute time for the VPARA solutions, even for 8000 structural steps ${ }^{*}$ for every VPARA fluid step. The structural time step is, however, very dependent on the spatial distribution of node points on the structure.

\section{Future Numerical Simulation Activities}

In FY98, we will initiate development of a 3-D Vortex Method code and companion 3-D fast solver for the teraFLOP computer, and we will begin coupling of the 3-D Vortex Method code to PRONTO. By the end of FY98, we will demonstrate a 3-D 100 million particle ( $10^{8}$ vortices) fast solver. The 3-D Vortex Method flow solver and its coupling to PRONTO will be completed in FY99. A trajectory module and a parachute deployment model will be added in FY99. By the end of FY99, we expect to demonstrate a 3-D, incompressible, coupled fluid/structure teraFLOP computational simulation of an inflating parachute. Modification

\footnotetext{
- 8000 structural steps per VPARA step is far more than will be used in the production Parachute Performance Code
} 
of the Vortex Method to take compressibility effects into account will begin as a research project in FY98-9 and be implemented into the Parachute Performance Code in FY00. In FY01, we will deliver a validated, documented, 3-D Parachute Performance Code for use on the teraFLOP computer.

\section{Code Validation Experiments}

Although Sandia has access to full-scale JTA parachute flight test data, these data are unable to validate the coupled fluid and structural dynamic computational models proposed for the ASCI Parachute Performance Code. We are in the process of conducting an experiment designed solely for the purpose of validating the fluid dynamic vortex model used in the axisymmetric, incompressible, bluff body unsteady parachute code.

This experiment uses a rigid, axisymmetric, hemispherical "ribbon parachute" model in a water tow tank. The primary variables being measured are time-dependent canopy drag and surface pressure distribution on the individual "ribbons."

Figure 4 shows the one-foot diameter experimental model being used in the experiment. Each of the four "ribbons" has pressure taps on its inner and outer surface to give differential pressures at five approximately equally spaced locations across the width of the ribbon. A similar pair of pressure taps are located at the center of each ribbon on the opposite side to provide assurance of axisymmetry. While the trailing struts appear to be intrusive, they are required both to provide structural integrity to the model and to provide a conduit to the data acquisition system for the 48 pressure lines. It is also felt that the "intrusiveness" of the struts is advantageous in that they stabilize the flow in the rotational plane in much the same way that a "splitter" plate reduces the tendency of a cylindrical shape to produce alternating yortices. Thus, the struts act to assure axisymmetric flow.

The experiments being run consist primarily of obtaining unsteady pressure data for various combinations of accelerating, steady, and decelerating flow. A nominal velocity profile is specified which the tow tank carriage attempts to replicate. The actual velocity profile is measured and used in the code validation computations.

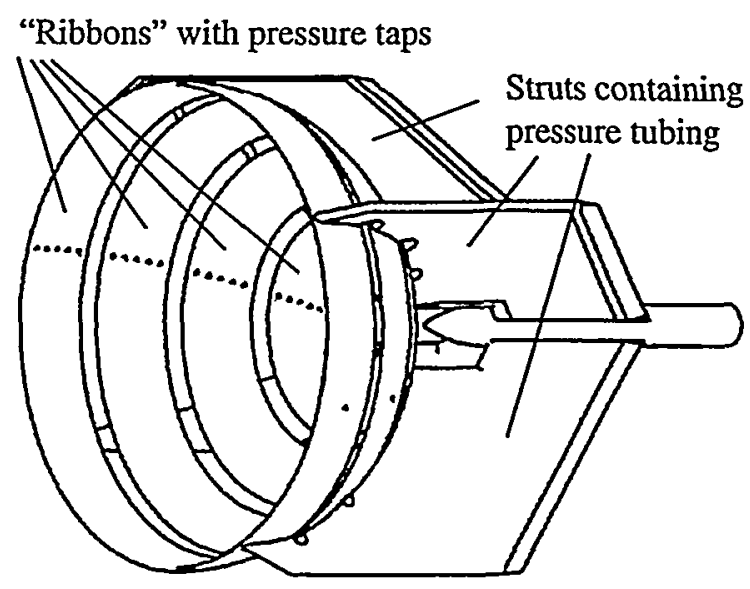

Figure 4. Water Tank Model

VPARA has been used to compute the wake flow, drag and pressure distribution around the four-ribbon tow tank model. The velocity time history consisted of an impulsive start ( 0 to $U_{0}$ in $\Delta t U_{0} / R_{0}=0.05$ ), after which the velocity was held steady at $U_{0}$. A snapshot of the wake geometry (regions of shed vorticity) is shown in Figure 5a for a non-dimensional time equal to 6.0 . The body has traveled 6.0 radial lengths $\left(R_{0}\right)$ during this time.

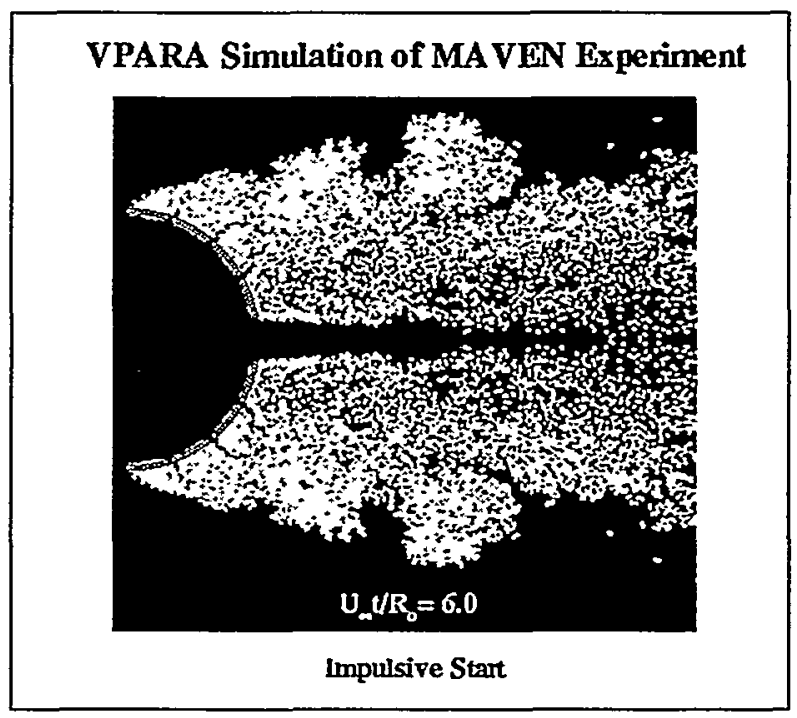

Figure 5a. VPARA Simulation of the Impulsively Started Four-Ribbon Tow Tank Model: Vortex Flow Field 
The differential pressure distribution $\Delta C_{p}$ on the four ribbons is shown at a non-dimensional time of 6.0 in Figure 5b. Here $s$ is the distance from the vent along the radial and $R_{0}$ is the radial length. In general, the pressures predicted from VPARA tend to be somewhat noisy due to the interaction of the vortices with the discrete collocation points. Since the differential pressure level is on the order of 1.4 for a hemisphere, the predicted pressure levels appear to be reasonable.

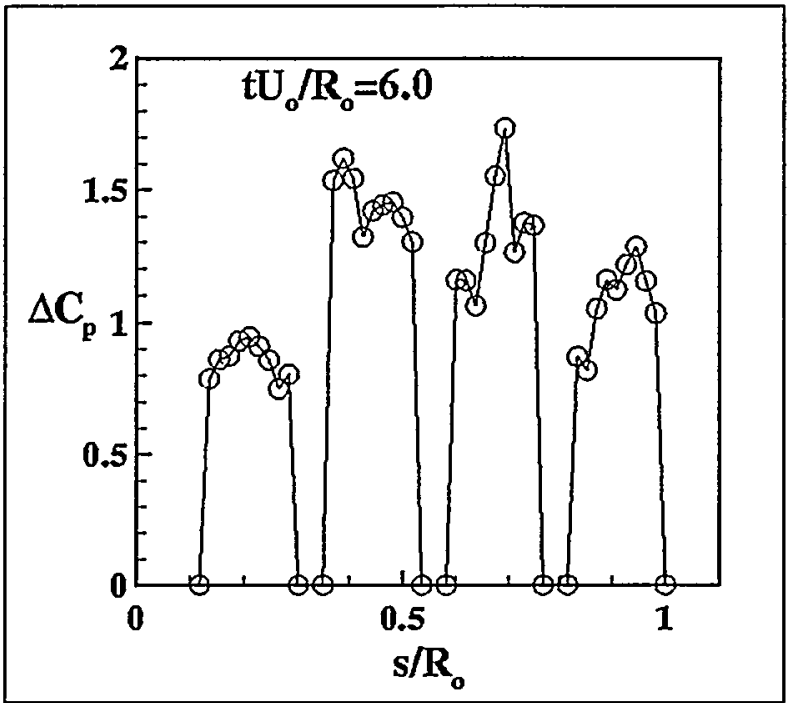

Figure 5b. VPARA Simulation of the Impulsively

Started Four-Ribbon Tow Tank Model:

Differential Pressure Distribution

The calculated drag time history is shown in Figure 5c. The drag coefficient $C_{D}$ is based on the area $\pi R_{0}^{2}$. An asymptotic value of about 0.4 is reached after about 4 radials of travel. The equivalent drag coefficient for a hemispherical shell is approximately 0.57 .

In subsequent years, we will propose experiments which focus on observing vortex shedding mechanisms, measuring the wake velocity field using a non-intrusive technique, venturing outside of the axisymmetric arena to validate the 3-D modeling of the vortices, and eventually attempting to measure the canopy dynamic and structural response to the unsteady bluff body flow field. It is obvious such experiments must increase in complexity and decrease in feasibility as the phenomena being replicated increase in complexity. It is only hoped that our knowledge and ingenuity in the design of such experiments might increase as well.

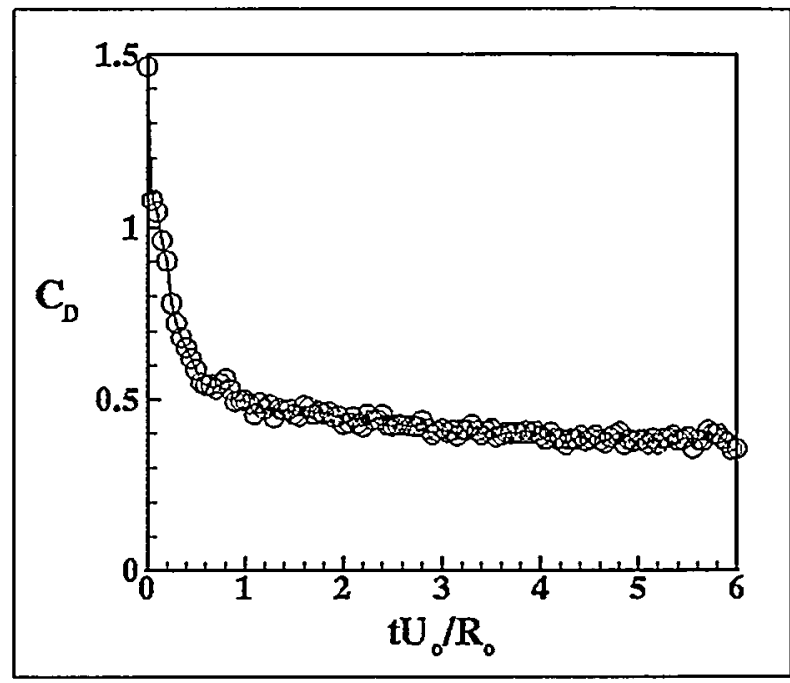

Figure 5c. VPARA Simulation of the Impulsively

Started Four-Ribbon Tow Tank Model:

Drag Coefficient History

\section{Program Direction}

In order to provide guidance of the Parachute Performance Code project, we desire to form an advisory committee comprised of parachute technologists and computational fluid and structural dynamicists. The committee will be utilized throughout the project to assure the relevance of the ASCI Parachute Prediction Code to the stockpile mission it is to serve. It will make recommendations on both computational and experimental tasks of this project.

\section{Summary and Conclusions}

Sandia National Laboratories has begun development of a parachute performance code to assist in the prediction of how stockpile weapon parachutes will perform during the remainder of their service lives. This code will utilize a massively parallel computer capable of performing over $10^{12}$ floating point operations per second using over 4500 pairs of Pentium Pro processors for concurrent calculations. Fluid dynamic calculations will be performed using a vortex model of the flow field, and canopy structural and kinematic calculations will be performed by the PRONTO large deformation finite element structural mechanics 
code. These codes will be coupled in order to calculate parachute inflation.

The axisymmetric vortex code VPARA uses the diffusion velocity concept to model viscous diffusion of vorticity from the parachute to the outer flow field. Although we currently believe that viscous effects can be ignored for bluff bodies like parachutes, we have prepared numerical algorithms to account for viscous effects in our vortex-based flow field solver in case our assumptions about the importance of viscosity in parachute aerodynamics prove incorrect. Good agreement between exact analytical solutions and VPARA predictions have been achieved for two laminar flow cases of Blasius flat plate flow and a sinusoidally oscillating flat plate.

Although we have just begun the development of the parachute performance code, we have identified several other computational and phenomenological issues that will require further investigation as our work progresses:

1. The choice of fast solver methodology for use with the vortex code may be determined as much by what method is most amenable to parallelization as by how well the fast solver reduces the size of the problem. Specifically, we are considering using a fast solver method which reduces the $O\left[N^{2}\right]$ problem of vortex interaction (where $\mathrm{N}$ is the number of vortices in the flow field) to $\mathrm{O}[\mathrm{NlnN}]$ because its computational efficiency on the massively parallel computer may be better than that of a fast solver which scales the problem to $O[N]$.

2. Coupling between the fluid dynamic vortex model and the structural/kinematic parachute model may prove to be more complex than a simple alternating fluid-step then structure-step approach. Because parachutes are lightweight structures, the normal acceleration of the apparent mass fluid force term may have to be included as part of the structural solution. However, the coupling should not require simultaneous solution of both the fluid and structural equations in the solution process.

Validation experiments are essential in order for the users of the Parachute Performance Code to have confidence in its predictions. An experimental validation program has been initiated to investigate the pressures on a parachute-like solid shape in a tow tank in accelerating and decelerating flow.

\section{Acknowledgement}

Sandia is a multiprogram laboratory operated by Sandia Corporation, a Lockheed Martin Company, for the United States Department of Energy under Contract DE-ACO4-94AL85000.

\section{$\underline{\text { References }}$}

1. Strickland, J. H. and Higuchi, H., "Parachute Aerodynamics: An Assessment of Prediction Capability (1995)," 13th AIAA Aerodynamic Decelerator Systems Technology Conference, Paper No. AIAA95-1531, May 1995.

2. Nelsen, J., "Computational Fluid Dynamic Studies of a Solid and Ribbon 12-Gore Parachute Canopy in Subsonic and Supersonic Flow," 13th AIAA Aerodynamic Decelerator Systems Technology Conference, Paper No. AIAA95-1558, May 1995.

3. Garrard, W. L., Tezduyar, T. E., Aliabadi, S. K., Kalro, V., Luker, J., and Mittal, S., "Inflation Analysis of Ram Air Inflated Gliding Parachutes," 13th AIAA Aerodynamic Decelerator Systems Technology Conference, AIAA 95-1565, May 1995.

4. Stein, K. R., Benney, R. J., and Steeves, E. C., "A Computational Model That Couples Aerodynamic and Structural Dynamic Behavior of Parachutes During the Opening Process," U. S. Army Technical Report NATICK/TR-93/029, February 1993.

5. Stein, K. R. and Benney, R. J. "Parachute Inflation: A Problem in Aeroelasticity," U. S. Army Technical Report NATICK/TR-94/015, February, 1994.

6. Sahu, J., Cooper, G., and Benney, R., "3-D Parachute Descent Analysis Using Coupled CFD and Structural Codes," 13th AIAA Aerodynamic Decelerator Systems Technology Conference, Paper No. AIAA95-1580, May 1995. 
7. Haug, E., Lasry, D., and Kermel, P., "Dynamic Simulation of Industrial Membranes Including Their Interaction with Surrounding Media," Third International Symposium of the SBF 230 Evolution of Natural Structures, University of Stuttgart, October 4-7, 1994.

8. McCoy, H. H., and Werme, T. D., "Axi-symmetric Vortex Lattice Method Applied to Parachute Shapes," AIAA 9th Aerodynamic Decelerator \& Balloon Technology Conference, AIAA 86-2456, pp. 140-146, Oct. 1986.

9. Strickland, J. H., "Prediction Method for Unsteady Axisymmetric Flow Over Parachutes," Journal of Aircraft, Vol. 31, No. 3, pp. 637643, May-June 1994.

10. Shirayama, S. and Kuwahara, K., "Computation of flow Past a Parachute by a ThreeDimensional Vortex Method," AIAA Paper No. 86-0350, AIAA 24 $4^{\text {th }}$ Aerospace Sciences Meeting, Reno, Nevada, January 1986.

11. Dneprov, I. V., "Computation of Aero-elastic Characteristics and Stress-Strained State of Parachutes," 12th RAeS/AIAA Aerodynamic Decelerator Systems Technology Conference and Seminar, AIAA 93-1237, pp. 240-244, May 1993.
12. Taylor, L. M. and Flanagan, D. P. "PRONTO: A Three-Dimensional Transient Solid Dynamics Program," Sandia National Laboratories Report SAND87-1912, March 1989.

13. Kempka, S. N. and Strickland, J. H., "A Method to Simulate Viscous Diffusion of Vorticity by Convective Transport of Vorticies at a Non Solenoidal Velocity," SAND93-1763, Sandia National Laboratories, December 1993.

14. Strickland, J. H., Kempka, S. N., and Wolfe, W. P., "Viscous Diffusion of Vorticity in Unsteady Wall Layers Using the Diffusion Velocity Concept," Forum on Vortex Methods for Engineering Applications, Albuquerque, NM, sponsored by Sandia National Laboratories, February 1995, pp. 69-83.

15. Strickland, J. H., Kempka, S. N., and Wolfe, W. P., "Viscous Diffusion of Using the Diffusion Velocity Concept," ESAIM: Proceedings, Vol. 1, 1996, pp. 135-151.

16. Schlichting, H.; Boundary Layer Theory, McGraw-Hill, pp. 125-129, 1968.

17. Curry, I. G., Fundamental Mechanics of Fluids, McGraw-Hill, 1974, pp.235-237. 